\title{
Early Components of the Human Vestibulo-Ocular Response to Head Rotation: Latency and Gain
}

\author{
HAN COLLEWIJN AND JEROEN B. J. SMEETS \\ Department of Physiology, Faculty of Medicine and Health Sciences, Erasmus University Rotterdam, 3000 DR Rotterdam, \\ The Netherlands
}

Received 12 November 1999; accepted in final form 6 April 2000

Collewijn, Han and Jeroen B. J. Smeets. Early components of the human vestibulo-ocular response to head rotation: latency and gain. $J$ Neurophysiol 84: 376-389, 2000. To characterize vestibulo-ocular reflex (VOR) properties in the time window in which contributions by other systems are minimal, eye movements during the first $50-100 \mathrm{~ms}$ after the start of transient angular head accelerations $\left(\sim 1000 \% \mathrm{~s}^{2}\right)$ imposed by a torque helmet were analyzed in normal human subjects. Orientations of the head and both eyes were recorded with magnetic search coils (resolution, $\sim 1 \mathrm{~min}$ arc; 1000 samples/s). Typically, the first response to a head perturbation was an anti-compensatory eye movement with zero latency, peak-velocity of several degrees per second, and peak excursion of several tenths of a degree. This was interpreted as a passive mechanical response to linear acceleration of the orbital tissues caused by eccentric rotation of the eye. The response was modeled as a damped oscillation $(\sim 13 \mathrm{~Hz})$ of the orbital contents, approaching a constant eye deviation for a sustained linear acceleration. The subsequent compensatory eye movements showed (like the head movements) a linear increase in velocity, which allowed estimates of latency and gain with linear regressions. After appropriate accounting for the preceding passive eye movements, average VOR latency (for pooled eyes, directions, and subjects) was calculated as $8.6 \mathrm{~ms}$. Paired comparisons between the two eyes revealed that the latency for the eye contralateral to the direction of head rotation was, on average, $1.3 \mathrm{~ms}$ shorter than for the ipsilateral eye. This highly significant average inter-ocular difference was attributed to the additional internuclear abducens neuron in the pathway to the ipsilateral eye. Average acceleration gain (ratio between slopes of eye and head velocities) over the first $40-50 \mathrm{~ms}$ was $\sim 1$.1. Instantaneous velocity gain, calculated as $V \mathrm{eye}_{t} / \mathrm{Vhead}_{t-\text { latency }}$, showed a gradual build-up converging toward unity (often after a slight overshoot). Instantaneous acceleration gain also converged toward unity but showed a much steeper build-up and larger oscillations. This behavior of acceleration and velocity gain could be accounted for by modeling the eye movements as the sum of the passive response to the linear acceleration and the active rotational VOR. Due to the latency and the anticompensatory component, gaze stabilization was never complete. The influence of visual targets was limited. The initial VOR was identical with a distant target (continuously visible or interrupted) and in complete darkness. A near visual target caused VOR gain to rise to a higher level, but the time after which the difference between far and near targets emerged varied between individuals.

\footnotetext{
Address for reprint requests: H. Collewijn, Dept. of Physiology, H-EE-1540, Faculty of Medicine and Health Sciences, Erasmus University Rotterdam, PO Box 1738, 3000 DR Rotterdam, The Netherlands (E-mail: collewijn @ fys.fgg.eur.NL).
}

\section{N T R O D U C T I O N}

Passive rotation of the head is accompanied, in species with mobile eyes, by compensatory eye rotation in the opposite direction such that gaze direction tends to remain relatively stable despite head movements. The earliest components of this ocular response (within $100 \mathrm{~ms}$ of the onset of head rotation) are controlled by the vestibulo-ocular reflex (VOR), which has the following characteristics: 1) the presence of very short connections (a three-neuron arc); 2) a common spatial organization between the sensory organ (the semi-circular canals) and the effector (the external eye muscles); and 3) sensitivity of the canals to rotational acceleration as the primary stimulus (see, e.g., Highstein 1988). These properties favor an early stabilization of gaze after sudden disturbances of the orientation of the head. Indeed, short VOR latencies have been reported: for monkeys, $14.2 \mathrm{~ms}$ (Lisberger 1984), $12 \mathrm{~ms}$ (Cullen et al. 1991), $10 \mathrm{~ms}$ (Snyder and King 1992), and $7.3 \mathrm{~ms}$ (Minor et al. 1999); for cats, $13 \mathrm{~ms}$ (Khater et al. 1993); for humans, 6-15 ms (Maas et al. 1989), 4-13 ms (Johnston and Sharpe 1994), 7-8 ms (Tabak and Collewijn 1994), and $10 \mathrm{~ms}$ (Crane and Demer 1998).

The action of the VOR in generating compensatory gazestabilizing eye movements is complemented by the optokinetic response (OKR), for which slippage of the retinal image is the primary stimulus. The OKR has a relatively long delay because the elaboration of visual motion signals requires considerably more signal processing than the VOR requires. The shortest latency described for optically driven compensatory eye movements in humans is 70-80 ms (Gellman et al. 1990). Any contribution to gaze stabilization in normal humans by proprioceptive cervico-ocular reflexes appears to be small and inconsistent (Bronstein and Hood 1986; Jürgens and Mergner 1989).

Thus the best strategy with which to investigate the VOR in a "pure" form is to measure ocular responses that occur within a window of $\sim 10-70 \mathrm{~ms}$ after the start of a transient, welldefined head movement. While the VOR in this early phase is unlikely to be affected by visual or propriocepive inflow that is directly derived from the ongoing head movement, it may still be modulated by factors that require a modification of the VOR gain, such as the distance of a visual target, the position of the axis of head rotation, or non-unity visual magnification factors. The topography of the axes of eye and head rotation requires an

\footnotetext{
The costs of publication of this article were defrayed in part by the payment of page charges. The article must therefore be hereby marked "advertisement" in accordance with 18 U.S.C. Section 1734 solely to indicate this fact.
} 
increase in VOR gain as a visual target gets nearer to the subject. Such a change has been demonstrated repeatedly (Biguer and Prablanc 1981; Blakemore and Donaghy 1980; Crane and Demer 1998; Hine and Thorn 1987; Snyder and King 1992; Snyder et al. 1992; Viirre and Demer 1996; Viirre et al. 1986) but its early time course is not well known.

Research on the human VOR has traditionally used wholebody motion with low-frequency sinusoidal oscillation or persistent rotation in one direction. Such long-lasting stimuli often yielded gain-values for the VOR that were substantially below unity and, moreover, subject to many extrinsic influences, such as mental frames of reference (for an overview see Collewijn 1989). Research with transient stimuli has been sparse, partly because of the technical limitations of the rotational devices used. Traditional human rotation devices do not generate accelerations much larger than $100 \% \mathrm{~s}^{2}$, but natural head rotations reach several times this magnitude during walking and running, and can be as high as $6000-12000 \% \mathrm{~s}^{2}$ during vigorous, voluntary head shaking (Grossman et al. 1988, 1989). Such high head accelerations can thus be considered physiological and apparently harmless. In some previous experiments, substantial acceleration pulses of the head alone were achieved. Maas et al. (1989) were able to determine the gain and latency of the human VOR by inducing head accelerations of up to $7100^{\circ} / \mathrm{s}^{2}$ by applying mallet strokes to a yoke that was clenched between the teeth of the subject. Halmagyi et al. 1990 and Aw et al. 1996 achieved head accelerations of up to $3000 \% \mathrm{~s}^{2}$ in manually applied passive steps in head orientation in normal subjects and in patients with vestibular disease. Such transient stimuli proved to be better tests of VOR performance than traditional motion stimuli but had the disadvantage of being relatively uncontrolled and variable.

In the last few years, some groups developed more powerful rotational devices for whole-body rotation of human subjects $\left[2800 \% \mathrm{~s}^{2}\right.$, Crane and Demer (1998); $284 \% \mathrm{~s}^{2}$, Johnston and Sharpe (1994)]. In a different approach, Tabak and Collewijn (1994, 1995) and Tabak et al. (1997a,b) introduced a torquedriven helmet to impose well-controlled transient head accelerations of about $1000 \% \mathrm{~s}^{2}$ with great facility. In the present experiments, the early phase of the normal human VOR in response to pulses of acceleration was investigated by using this device with improved recording and analysis procedures, binocular recording, and a number of different target distances and visibility conditions. In particular, the latency and early build-up of acceleration gain and velocity gain were addressed, as well as the occurrence of mechanical ocular responses in the latency period.

\section{METHOD S}

\section{Subjects}

Healthy subjects without any known vestibular or oculomotor abnormalities were recruited after informed consent. The procedures were approved by the Medical Ethical Committee of the Faculty of Medicine. A few subjects were rejected because they tended to blink in association with the head stimuli, which introduced unmanageable artifacts into their recordings. Ten subjects were retained for analysis; some analyses and comparisons were made in smaller subsets of these subjects.

\section{Motion stimuli}

The use of the torque helmet was described previously (Tabak and Collewijn 1994, 1995). In the present experiments, acceleration pulses in the horizontal plane were delivered by activating the torque motor for $200 \mathrm{~ms}$ at maximum power. These pulses were alternated in the rightward and leftward directions. The interval between pulses was randomized between 2.5 and $3.5 \mathrm{~s}$ (average, $3 \mathrm{~s}$ ); one measuring sequence lasted $180 \mathrm{~s}$. Thus $\sim 30$ pulses in each direction were delivered in one measuring sequence. The subjects, while wearing the helmet, were not rigidly attached to any fixed structure and were relatively free to orient and move their heads.

\section{Visual stimuli}

Seven visual conditions were tested. The first measurement was done in complete darkness. In the other six conditions, a single red light-emitting diode (LED) was presented at two distances, $\sim 220$ or $\sim 40 \mathrm{~cm}$, and in three conditions of visibility. The LED was extinguished 50 or $500 \mathrm{~ms}$ before the activation of the helmet or was left on throughout the measurement.

\section{Eye movement recording}

Movements of both eyes were recorded with the scleral coil technique (Robinson 1963). Coils embedded in a silicone annulus (Skalar, Delft, The Netherlands) were inserted in each eye (Collewijn et al. 1975). A Remmel EM3 eye-movement recorder (Remmel Labs, Ashland, MA) was adapted for large, earth-fixed field coils (pairs of square coils; diameter, $2.5 \mathrm{~m}$; inter-coil distance, $1.25 \mathrm{~m}$; this provided a "Helmholtz" coil configuration with sufficient homogeneity). Head movements were recorded by a third coil, which was mounted to an individually molded silastic dental-impression bite-board. All coils were pre-calibrated on an angular rotation device. Gains and offsets of the instrument were extremely stable. It was verified that calibrations were unaffected by translations of the coils over a range (up to $20 \mathrm{~cm}$ in all directions) that exceeded any spontaneous head displacements by the subjects. The noise level corresponded to $<1$ min arc at a recording range of $20^{\circ}$ on each side of the middle position. The resulting signals represented the orientation of the head in space and the eyes in space (gaze).

\section{Data collection and analysis}

Orientations of the eyes and head were sampled at a frequency of $1010 \mathrm{~Hz}$ (each channel) with a CED 1401-plus AD-converter with the CED Spike 2 program (Cambridge Electronic Design Ltd., Cambridge, UK) and stored on disk. The same device was programmed to generate the pulses that controlled the torque helmet, and marker signals indicating the timing of these pulses were included in the recordings. In the subsequent off-line analysis, angular position signals were converted to angular velocity signals by digital differentiation using five subsequent samples without time shift (see Collewijn et al. 1995). This routine eliminated much of the noise at the cost of a mild time-blurring due to smoothing two position samples forward and backward. Accelerations were calculated by differentiating velocity. The larger noise inherent to this procedure necessitated the use of nine subsequent velocity samples with, as a consequence, more time blurring (smoothing four velocity samples and, therefore, six position samples forward and backward). After removal of the (occasional) events that were contaminated by blinks or saccades at critical moments, the responses in a measurement sequence were averaged for each direction separately; temporal alignment was achieved by a computer-generated trigger locked to the electrical command to the helmet. Eye-in-head movements were computed by subtracting head movements from gaze movements; vergence and version were computed as the difference between and the average of the orientations of 
the two eyes. Statistical differences were tested with paired $t$-tests whenever appropriate.

\section{R E S ULTS}

\section{The basic response}

The basic result is presented in Fig. 1, which shows head angular velocity and eye angular velocity (the latter is shown inverted for clarity, i.e., head velocity - gaze velocity) as a function of time for a typical subject $(M F)$. These results were obtained with a distant visual target that was extinguished 50 ms prior to the head stimulus. Figure $1 A$ shows 10 subsequent individual head and eye movements superimposed, to show the reproducibility of all main components. Figure $1 B$ shows the average head and eye velocities for the same measurement, which is composed of 23 consecutive head acceleration pulses in the same (rightward) direction. Standard deviations of head and eye velocities (shown as vertical gray bars) were small at all times; the movements were very reproducible within a measurement (which lasted $3 \mathrm{~min}$ ) with very little variability between impulses. The further analysis of our data is based on such averages of all (uncontaminated) responses $(n=20-30)$ in a measurement.

Head acceleration built up over an initial period of $\sim 10 \mathrm{~ms}$
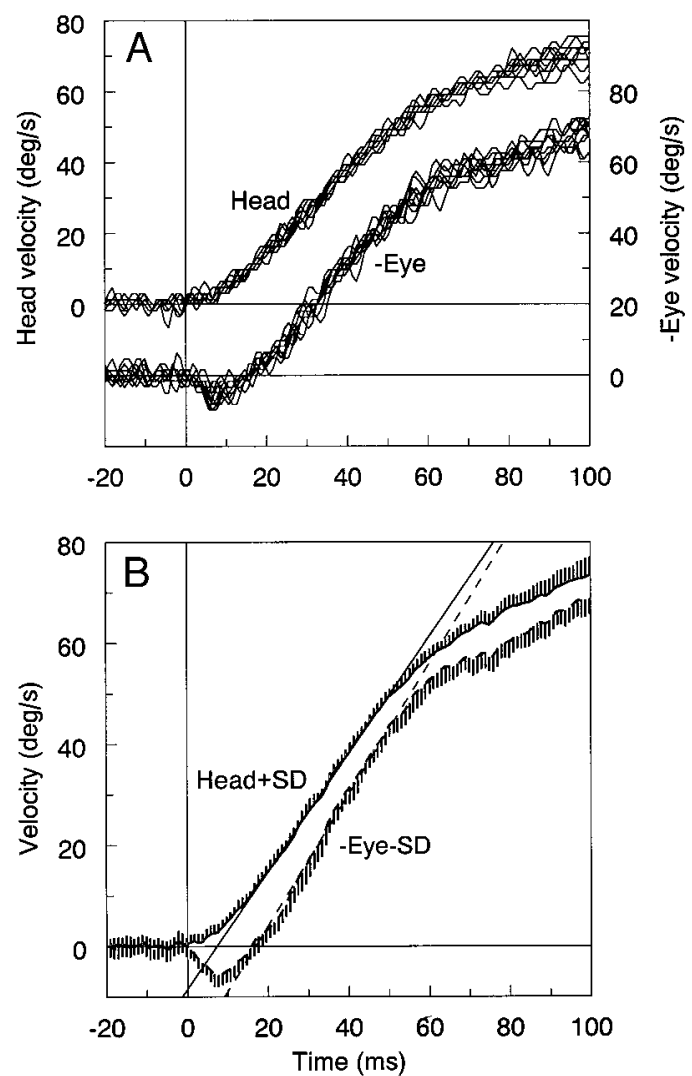

FIG. 1. Typical examples (subject $M F$ ) of head and right eye velocities elicited by a rightward pulse of head acceleration starting at time 0. A: 10 subsequent individual pulses superimposed to show noise level and variability within a measurement. Eye velocity has been inverted for easy comparison with head velocity. $B$ : mean $\pm \mathrm{SD}$ (vertical gray bars) of 23 similar rightward rotations in the same measurement as in $A$. Such averages from a measurement formed the basis of all subsequent analysis. Linear regressions on head and eye velocity over a range of $10-50 \% \mathrm{~s}$ are shown. Visual condition: 1 light-emitting diode (LED) at $220 \mathrm{~cm}$ distance extinguished $50 \mathrm{~ms}$ before head movement. to steady values of $1000-1200^{\circ} / \mathrm{s}^{2}$ (depending on the subject) that were then maintained for 40-50 ms, during which time velocity rose approximately linearly as a function of time. Later, head velocity tended to saturate smoothly despite the continued force exerted by the helmet (which lasted for 200 $\mathrm{ms}$ ). This decrease in head acceleration (the exact course of which varied between individuals) may be attributed to the buildup of passive and active mechanical resistance during progressive rotation of the neck. The present analysis is essentially limited to the period with (approximately) constant head acceleration.

Eye velocity approximately mirrored head velocity, showing a similar time course, but separated along the time axis by a delay that was maintained over time. In a majority of the subjects, however, the shape of the early VOR was complicated by the occurrence of an anti-compensatory eye rotation that preceded the compensatory VOR and started at the same time as the head movement. Typically, such early anti-compensatory eye movements reached peak velocities of several $\%$, displacements of several hundredths of a degree, and accelerations of several hundred $\% \mathrm{~s}^{2}$ (Fig. 1). Their mean peak velocity was $3.26^{\circ} \pm 3.17^{\circ}(\mathrm{SD})$ for far targets and $3.60^{\circ} \pm$ $3.06^{\circ}$ (SD) for near targets (pooled results of 6 subjects, 2 eyes, 2 directions, and $2 \times 3$ visibility conditions; difference not significant in paired $t$-test). The manifestation of this anticompensatory component with zero latency relative to the head movement, and its apparent duration commensurate with the probable latency of the active VOR, strongly suggests a passive mechanic origin. We will further analyze its nature in The nature of the anti-compensatory eye movement after proceeding first with calculations of VOR latency and gain.

The occurrence of periods of relatively constant acceleration of head and eyes allowed a simple analytical procedure for determining the latency and initial gain of the VOR, as illustrated in Fig. 1B. Linear regressions were fitted to the straight parts of the velocities of the head, each of the eyes separately, and the two eyes combined (average velocity of the two eyes is called version). The later parts, in which acceleration declined, as well as the earliest parts, during which acceleration usually showed a short buildup and the eyes moved in the anti-compensatory direction, were not included in the regressions. In general, head and eye velocities were regressed over a range of $10-50 \%$ s (time span $\sim 15-50 \mathrm{~ms}$ after the start of the head movement). These ranges were individually adjusted whenever visual inspection of the velocity graphs revealed a different range of the straight parts of the velocity profiles. The coefficient of determination $\left(r^{2}\right)$ of the linear regressions was typically $\sim 0.99$. Each regression was characterized by its intersection with the time axis and its slope; the relation between these parameters for the head- and eye-velocity regressions yield independent estimates for VOR gain and latency. Gain is estimated as the ratio between the slopes of the linear regressions of eye and head velocity; because these slopes represent acceleration, this estimate reflects the acceleration gain. Latency is the time interval between the intersections of the linear regressions with the time axis. This technique for estimating latency is similar to that used by Carl and Gellman (1987) for the estimation of smooth-pursuit latencies and by Johnston and Sharpe (1994) for the VOR. 


\section{Acceleration gain}

The distribution of acceleration gain values, obtained as described in The basic response, is shown in Fig. 2 for the pooled data of six subjects for whom complete data were collected for two directions and seven conditions. First, it was established that the variations in the visibility of the target during head movement (switched off 50 or $500 \mathrm{~ms}$ prior to the stimulus or left on) did not have any systematic effect on the responses in the early period that we analyzed. Accordingly, the results for the three different visibility conditions were pooled for the near and far target. Next, we tested for differences in gain between distant and near target conditions. A paired $t$-test confirmed that gain was significantly higher (as was theoretically expected, $P=0.04$ ) for near targets than for far targets. Therefore separate histograms were plotted for near and far targets. For far targets, the mean early acceleration was 1.089 (Fig. 2A) whereas for near targets it was 1.124 (Fig. 2C). In darkness, mean gain was similar to that with a distant target (mean, 1.09; Fig. 2E). Ideally, steady-state gain values would be $\sim 1.045$ for the far target and $\sim 1.25$ for the near target. As will be elucidated in Instantaneous VOR gain, the early gain values do not reflect a steady state and should not be expected to correspond to these ideal values. It can be concluded that in an early period $(\sim 15-50 \mathrm{~ms}$ after the start of the head movement) VOR gain shows some systematic tendencies: 1) gain is larger for near than for distant targets; 2) all gains $(a / a)$ are systematically larger than unity.

A further differentiation was a comparison of the gain of the ipsilateral eye (the eye on the side to which the head rotated) with the gain of the contralateral eye. A systematic difference could occur for two possible reasons. First, there could be an intrinsic difference in the VOR dynamics for nasal and temporal eye movements, like there is for horizontal "conjugate" saccades in which the abducting eye usually reaches a higher peak velocity than its fellow eye. This would allow us to predict a higher gain of the contralateral (abducting) eye for the VOR. Second, differences in gain could result from the difference in distance of the two individual eyes to the target as a function of head position. Inter-ocular differences of this type should emerge, especially for near targets (Viirre et al. 1986). Paired $t$-tests comparing gains of the contralateral and ipsilateral eye were done for the different target conditions. For far targets, the mean difference in gain (ipsilateral eye gain contralateral eye gain $=0.014$ ) was not significantly different from zero $(P=0.35$; see distribution in Fig. $2 B)$. For near targets, the mean ipsilateral gain was significantly higher than the contralateral gain (difference of $0.123 ; P=3.7 \times 10^{-5}$; see Fig. 2D). In darkness, there was again no difference (mean $0.013 ; P=0.55$; see Fig. $2 F)$.

To interpret the inter-ocular gain difference for the near


FIG. 2. Distributions of acceleration gains derived from linear regressions (slope eye velocity/ slope head velocity) for a far target $(A)$, a near target $(C)$, and darkness $(E)$. Pooled results from 6 subjects, 2 eyes, 2 directions, and (for $A$ and $C$ ) 3 visibility conditions of targets (extinguished at -50 or $-500 \mathrm{~ms}$ or left on). Differences between the gains of the ipsilateral and contralateral eyes (relative to the direction of rotation) are shown in $B, D$, and $F$. The difference is statistically significant only for the near target. 

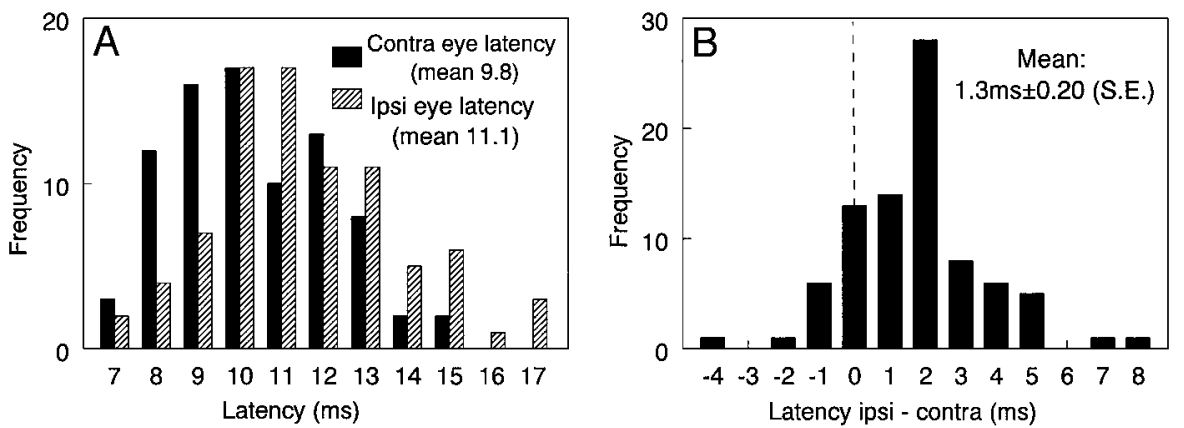

FIG. 3. A: distribution of vestibulo-ocular reflex (VOR) latency for the contralateral and ipsilateral eyes estimated from linear regressions on eye and head velocity. Pooled data of 6 subjects, 7 visual target conditions, and 2 directions. $B$ : distribution of the difference between the latency of the ipsilateral and contralateral eyes. targets, the initial head position has to be known. If the head is rotated to the right from an initial angular position that is to the left of the middle, the ipsilateral (right) eye should have a higher initial VOR gain because it is closer to the target than the left eye (Viirre et al. 1986). This was actually the case in our experiments. The alternation of rightward and leftward pulses had the result that the mean initial positions of the head were $\sim 3^{\circ}$ left of the middle position for rightward pulses that were preceded by leftward pulses, and vice versa. The fact that the inter-ocular gain difference virtually disappeared with far targets and darkness supports the hypothesis that it originates in different eye-target distances and argues against an intrinsic advantage for nasal or temporal VOR movements. In particular, there was no evidence for any advantage of the contralateral (abducting) eye, as occurs in saccades.

\section{VOR latency}

Although there is no reason to expect differences in VOR latency due to visual target conditions, the presence of a minimum of three and two synapses in the shortest VOR pathways to the medial and lateral rectus muscles, respectively, suggests a possible shorter latency of the contralateral than of the ipsilateral eye. A paired $t$-test corroborated the absence of a significant difference $(P$ two-tailed $=0.10)$ between the mean latency for far targets $(10.3 \mathrm{~ms})$ and near targets $(10.8$ $\mathrm{ms}$ ). Accordingly, all conditions (far and near targets and darkness) for the six completely measured subjects were pooled for a comparison between the latency estimates for the ipsilateral and the contralateral eyes. The two distributions, as determined from the intersections of the regression lines on eye and head velocity with $V=0$, are presented in Fig. 3A. These histograms show that latency was systematically longer for the ipsilateral than for the contralateral eye. For statistical analysis, the two eyes were paired for comparison within a measurement, i.e., for head pulses to the right the latency of the right eye (ipsilateral) was compared with the latency of the left eye (contralateral) and vice versa. Mean values were $11.1 \pm 0.2 \mathrm{~ms}$ (SE) and $9.8 \pm 0.2 \mathrm{~ms}(\mathrm{SE})$; the difference was statistically very significant (two-tailed paired $t$-test, $P=5 \times 10^{-9}$ ). The distribution of the difference (mean value $1.3 \pm 0.2 \mathrm{~ms}, \mathrm{SE}$ ) is shown in Fig. $3 B$.

The shorter mean latency for the contralateral eye than for the ipsilateral eye was also present in each of the seven stimulus conditions separately. However, it could not be demonstrated at the level of individual measurements, probably as a result of interference by the anti-compensatory component, which showed random variations between eyes and measurements, thus masking the subtle systematic differences in eye latencies. Given the statistical robustness of the effect for the pooled data, a grand average of the VOR of the contra- and ipsilateral eyes (6 subjects, 7 conditions, and 2 directions, normalized for rightward head rotation) is shown in Fig. 4, which shows the consistent delay of the response of the ipsilateral versus the contralateral eye by $\sim 1 \mathrm{~ms}$, which is maintained over time. Figure 4 also prominently shows the early anti-compensatory eye movement in the pooled data.

\section{Graded effects of anti-compensatory eye movement on latency and gain}

The magnitude of the anti-compensatory early eye movement differed systematically among subjects and, furthermore, randomly between eyes, directions, and measurements at different times. The latency and gain measurements from linear regressions as described in VOR latency and Acceleration gain disregard the anti-compensatory eye velocity attained during the latent period and assume implicitly that the active eye movement starts, like the head movement, from a velocity of zero. Actually, the active VOR is likely to start before the anti-compensatory movement has dissipated, i.e., while eye velocity is negative. In other words, the appropriate reference level for the start of the VOR is not zero eye velocity but a negative eye velocity. This would imply that the latencies as estimated in VOR latency tend to be overestimates; apparent latency is likely to increase as a function of the magnitude of the anti-compensatory component. If this is the case, then there should be a correlation between the latencies and the magnitude of the anti-compensatory movements in individual measurements. Figure $5 A$ shows the relation between measured

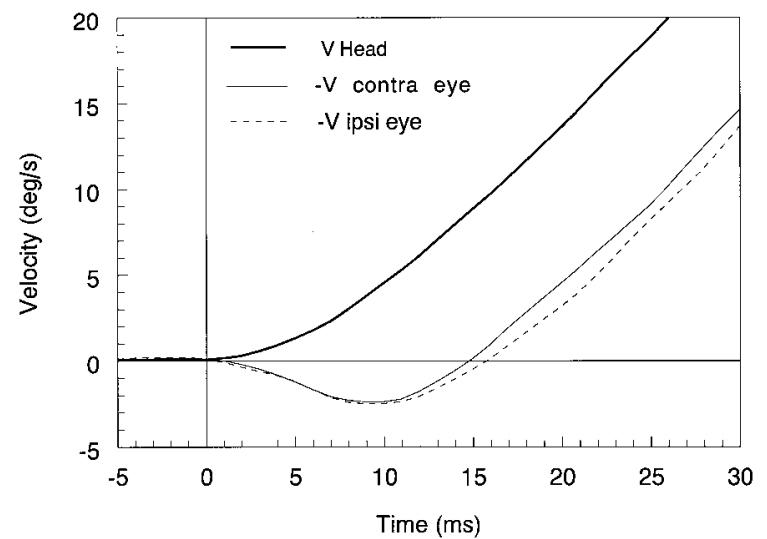

FIG. 4. Speed profiles of the head and the contralateral and ipsilateral eyes. Pooled data of 6 subjects, 7 conditions, and 2 directions (here normalized to rightward) showing the average latency difference between the eyes. 

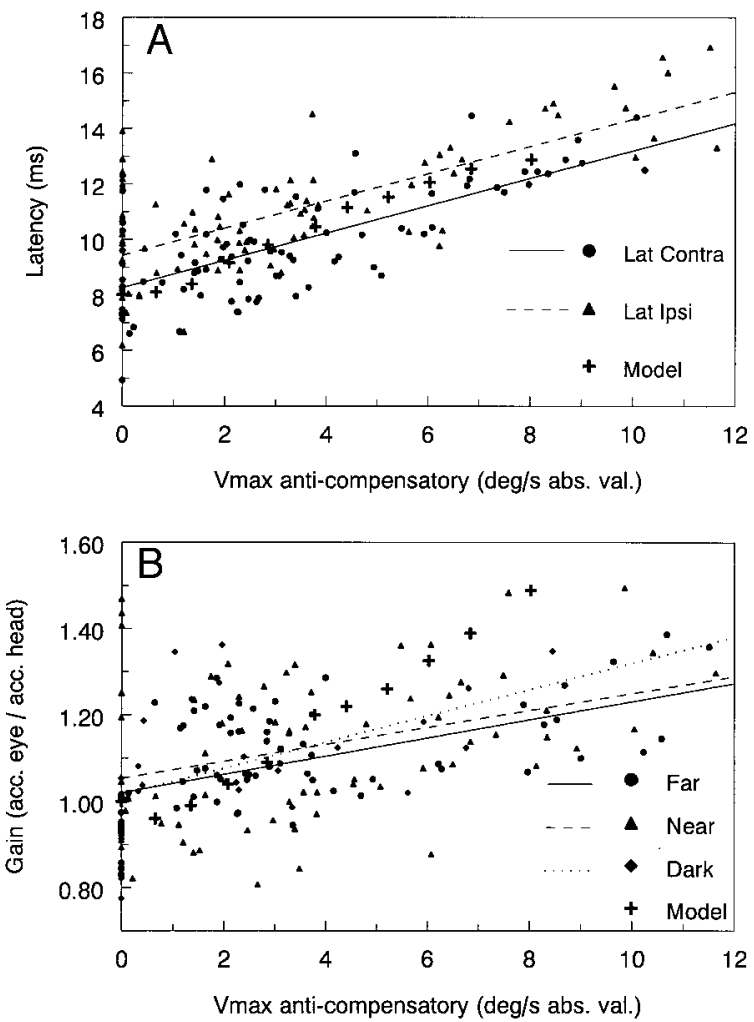

FIG. 5. Scatter plots of latency and gain as a function of the absolute value of the maximum anti-compensatory velocity. Pooled data for 6 subjects, 2 directions, 2 eyes, and 7 conditions (distant or near target and darkness). A: latency estimates showing the apparent increase in latency as a function of increasing anti-compensatory speed. The two separate linear regressions were calculated for the data representing the contralateral and ipsilateral eyes. $B$ : similar tendency for acceleration gain to increase with increasing anti-compensatory velocities. Three separate linear regressions were calculated for far and near targets and darkness. The crosses represent model data, as described in Isolation and modeling of the passive eye movements.

latencies and the absolute values of the maximum anti-compensatory velocity in individual measurements (i.e., averages of 20-30 successive head pulses). Because there was no statistical latency difference between visual conditions, all measurements of the six complete subjects were again pooled but the data for the ipsilateral and contralateral eyes were treated separately. Peak anti-compensatory eye speeds ranged from 0 to $\sim 12 \%$; latency increased as a function of the maximum anti-compensatory velocity. Separate linear regressions were done for the ipsilateral and contralateral eyes. Both accounted for about half of the variability $\left(r^{2}=0.53\right.$ and 0.49 , repectively) and showed a perfectly parallel course [slope $=0.49$ $\mathrm{ms} /\left(\operatorname{deg} \times \mathrm{s}^{-1}\right)$ ]. They were therefore separated by a constant time difference corresponding to the difference in latency between the ipsilateral and contralateral eyes. The intercepts of the regressions with the latency axis (i.e., for an anti-compensatory velocity equal to zero) were $8.25 \mathrm{~ms}$ for the contralateral eye and $9.43 \mathrm{~ms}$ for the ipsilateral eye. We postulate that these figures are the best estimates of the true latency of the active VOR that can be reached in the absence of detailed knowledge of the passive anti-compensatory component that would allow calculation of its exact contribution to the apparent latency. The difference between the intercepts $(1.18 \mathrm{~ms})$ agrees well with the estimate reached in Fig. $3 B$ for the mean difference in individual measurements $(1.3 \mathrm{~ms})$.
Figure $5 B$ shows a similar exercise for gain values. Linear regressions were calculated for three subgroups of the data: far target, near target, and darkness (with ipsilateral and contralateral eyes pooled together). For all groups, estimated gain increased as a function of the magnitude of the anti-compensatory response. The intercept of the regressions with the gain-axis (absence of anti-compensatory movement) was 1.02 for the "far" and "dark" conditions whereas it was higher (1.05) for the "near" condition. The difference $(0.03)$ was in good agreement with the difference $(0.035)$ between the mean gains for the populations (cf. Fig. 2, $A$ and $C$ ). Again, we postulate that these intercept values are the best estimates for the early VOR gain (averaged over a period of $\sim 15-50 \mathrm{~ms}$ after the start of the head movement) whereas the higher apparent values are a side effect of the anti-compensatory eye movement.

\section{The nature of the anti-compensatory eye movement}

The appearance of anti-compensatory eye movements simultaneously with the start of the head rotation, i.e., in the latency period of the active VOR, strongly suggests that the anticompensatory movement is mechanical, not neural, in nature. After carefully considering the possibility of, but not finding, any plausible errors of measurement, we assumed that the anti-compensatory movement originates from forces acting directly on the eye. It cannot be explained, however, as a reaction purely to the rotation of the head; any mechanical response of the eye to head rotation would have to be in the compensatory direction because of inertia of the eye in the orbit that undergoes a rotational acceleration. The same would apply to any inertial movement of the coil relative to the eye. It is difficult to predict the theoretical magnitude and, especially, the dynamics of an ocular inertial rotational response because the eye is not a rigid body but a fluid-filled shell. An attempt to calculate the theoretical mechanical compensatory eye movement during the first $10 \mathrm{~ms}$ of an angular head acceleration of $3000^{\circ} / \mathrm{s}^{2}$ yielded a magnitude of $0.002^{\circ}$ (Minor et al. 1999), which is clearly below the resolution of current recording techniques. However, the mechanical relations are complicated by the fact that the passive rotation of the eye is eccentric. As illustrated in Fig. 6A, rotation of the head around its natural axis of rotation near its center $(\mathrm{H})$, as imposed by the helmet, causes an eccentric rotation of the eye (and orbit) with radius $r$. A rotational acceleration (arot, expressed in radians/ $\mathrm{s}^{2}$ ) around $\mathrm{H}$ will induce a linear acceleration (alin, expressed in $\mathrm{cm} / \mathrm{s}^{2}$ ) at eccentricity $r$ (expressed in $\mathrm{cm}$ )

$$
a \operatorname{lin}=a \operatorname{rot} \times r
$$

At eye level, our angular accelerations would induce a linear component on the order of $0.2 \mathrm{~g}$ ( $r$ being $\sim 8-10 \mathrm{~cm}$ ). (Centripetal acceleration originating from the rotation is so small, on the order of $10^{-3} \mathrm{~g}$, as to be negligible in the first $10 \mathrm{~ms}$ of rotation.) At first sight, such a linear, laterally directed acceleration might not seem to affect the angular position of a ball-shaped body with its own center of rotation, such as the eye. In truth, however, the mechanical relations of the eye are very different. The eye and its surrounding tissues (muscles, membranes, ligaments, etc.) are an assembly of soft materials (the orbital contents) that are encased in a stiff bony box, the orbit. This box is not closed but open at the frontal side, where 


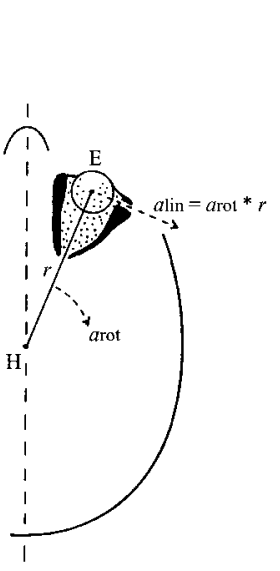

A

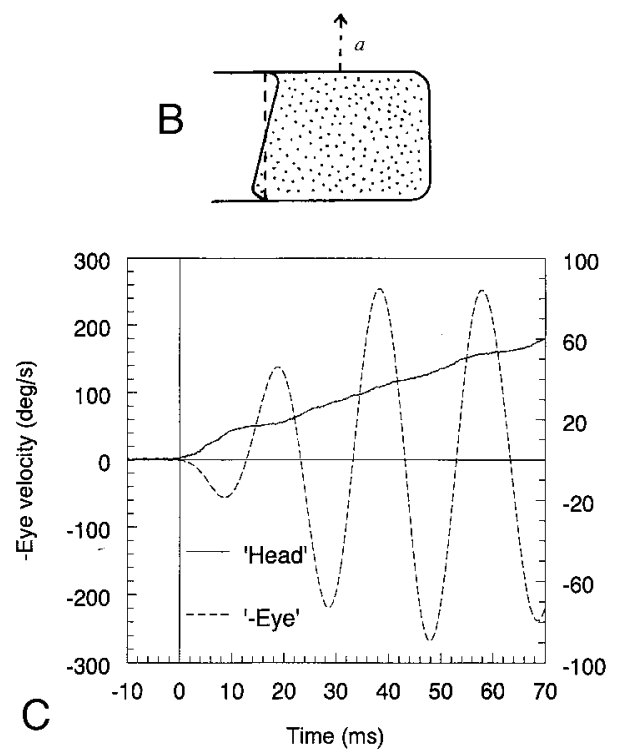

FIG. 6. The mechanical relations of the eye and its surrounding tissues in the orbit. $A$ : the emergence of a linear acceleration of the orbit as a result of eccentric rotation. $B$ : a semi-solid gel in a rigid vessel as the mechanical analogue of the tissues in the bony orbit. A linear acceleration parallel to the free surface causes a deformation that corresponds to an anti-compensatory rotation. $C$ : a dummy experiment with eccentric rotational acceleration of the device in $B$ instead of a subject's head. Coils were attached to the bottom of the vessel ("head") and the free surface ("eye"). The eye velocity was initially anti-compensatory; subsequently it oscillated. tissues are in contact with the air. Thus we have to consider the effect of a linear acceleration on such a structure. Although the architecture and mechanical properties of the orbital tissues may appear to be so complex as to preclude such an analysis, it turns out that the first-order effect can be modeled quite simply.

Consider the following mechanical analogue of the orbital tissues in the bony orbit: a glass beaker filled with a semi-solid gel (Fig. 6B; a fluid-filled beaker closed at the open surface with an elastic membrane would be equivalent). When this vessel is tilted to a horizontal position to align gravity with the free surface, or when the vessel is linearly accelerated in a direction parallel to the free surface, pressures in the gel will force the beaker's contents in the direction opposite to the acceleration. The result is a deformation of the surface, with its center rotating in the direction of the acceleration (Fig. 6B). For the head and eye (Fig. 6A), this effect corresponds to an anti-compensatory eye rotation.

To simulate this effect in our experimental conditions, we filled a small glass beaker $(50 \mathrm{ml}, 3.5 \times 6 \mathrm{~cm})$ with a warm $6.5 \%$ solution of gelatin and floated an eye coil on the surface. A second coil was glued to the outside of the bottom of the beaker; this mimicked the "head" coil. After the gel solidified and the "eye" coil became embedded in its free surface, the beaker was tilted to a horizontal position in the magnetic field (in two opposite directions) to assess the static steady-state effect of gravity. This effect was in the direction sketched in Fig. $6 B$ and its magnitude was $\sim 2^{\circ}$. The beaker was then mounted horizontally on a rotational device with the free surface at $10 \mathrm{~cm}$ eccentricity; this assembly was coupled to the torque helmet. A dummy experiment was then run, with the beaker undergoing dynamic accelerations similar to those applied to the orbits of our subjects, while the angular positions of the beaker ("head") and the free gel surface ("eye") were recorded. After data processing identical to that in the real experiments, results were obtained as shown in Fig. $6 C$. "Head" velocity accelerated almost uniformly to $60 \%$ s after 70 ms. "Eye" velocity started (with zero latency) in the anticompensatory direction. Velocity remained negative for $\sim 12$ $\mathrm{ms}$ but, instead of simply regressing to zero (corresponding to a steady deformation), it showed strong oscillations. This is actually not very surprising because our gelatin analogue represents an elasticity-viscosity-mass system that is unlikely to be critically damped. If acceleration were to be maintained for sufficient time, oscillations would decay and the deformation would reach a steady state, representing an equilibrium between the pressures caused by the acceleration and the elasticity of the deformed material. The primary conclusion at this point is that the early anti-compensatory eye rotation is easily accounted for (at least qualitatively) by a fundamental physical effect.

\section{Isolation and modeling of the passive eye movements}

The combined results described in The nature of the anticompensatory eye movement suggest that the eye movements observed in our experiments are the result of two processes: 1 ) a passive mechanical response to a step in linear acceleration caused by eccentric rotational acceleration of the orbit; 2) an active, neurally mediated VOR. Presumably, these processes simply add up to the total eye movement

$$
\text { Veye }=\text { Vpas }+ \text { Vact }
$$

This led us to the following analysis. Our data (Fig. 5) indicate that the active VOR has a gain very near unity and a delay of $\sim 8 \mathrm{~ms}$. On this basis, we took the data from a subject with a distinct anti-compensatory early eye movement and reconstructed the theoretical active VOR simply as a copy of the head velocity (gain $=1$ ) delayed by $8 \mathrm{~ms}$

$$
\operatorname{Vact}_{t}=- \text { Vhead }_{t-0.008}
$$

Next, we subtracted this theoretical active response from the recorded eye movement to retain an approximation of the passive eye velocity. A result is shown in Fig. 7A. Surprisingly, the reconstructed passive movement showed oscillatory behavior qualitatively similar to the gelatin model in Fig. $6 C$. This result was confirmed for other subjects; all showed passive oscillations with a frequency on the order of 12-15 Hz. Integration of the passive velocity in Fig. $7 A$ over time yielded passive eye position (Fig. $7 B$ ). This figure shows that, after a 

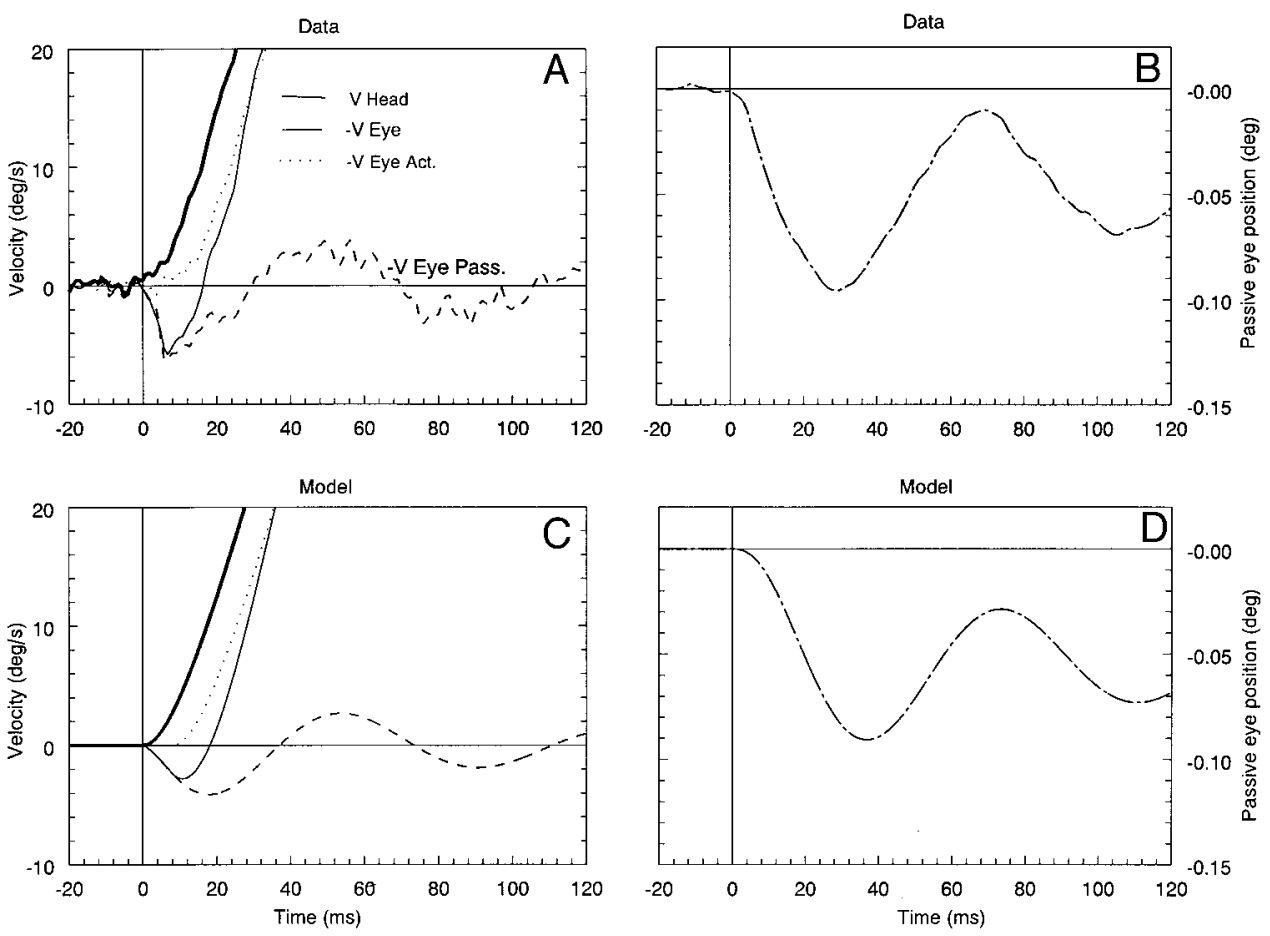

FIG. 7. A: reconstruction (using real data) of the passive mechanical eye response by subtracting a theoretical active VOR with a gain of 1 and a latency of $8 \mathrm{~ms}$ from the recorded eye movement. The result is an initially anti-compensatory eye velocity that is followed by oscillation (qualitatively resembling Fig. 6C). B: the passive mechanical response, expressed in angular position, obtained by integrating the reconstructed mechanical velocity response in $A$. $C$ and $D$ : as in $A$ and $B$, but for model data simulating the eye movements as the sum of a passive and an active component, as described in Isolation and modeling of the passive eye movements.

step in linear acceleration, the eye approached a steady deviation with a damped oscillation. Such a damped oscillatory change in position is described by the following equation

$$
y_{t}=y_{a}-y_{a} e^{-t / \tau}[\cos (\omega t)+\sin (\omega t) /(\tau \omega)]
$$

in which $y_{t}$ is position at time $t$ after the acceleration step, $y_{a}$ is the asymptotic end position that will be reached under a constant acceleration, $\tau$ is the time constant of the decay of the oscillation, and $\omega$ is the frequency of oscillation ( $\mathrm{rad} / \mathrm{s})$.

To mathematically model the eye movements observed, Eq. 4 was computed over an appropriate time range with a spreadsheet program and the corresponding passive velocity (Vpas) and acceleration (Apas) were obtained by differentiation. Equation 4 models a pure step response, which corresponds to an instantaneous rise of the angular head acceleration to $\sim 1000 \% \mathrm{~s}^{2}$. The actual head accelerations did not rise instantaneously; their behavior was well-modeled by an exponential rise to the maximum value with a time constant of $0.01 \mathrm{~s}$. Accordingly, head velocities rose initially smoothly before reaching the period of constant acceleration. These features were implemented in the models of the head movements and the passive eye movements by letting $y_{a}$ rise to its asymptotic value with a time constant of $10 \mathrm{~ms}$. Active VOR eye velocity (Vact) was modeled by Eq. 3 and total eye velocity by Eq. 2 .

A typical result is shown in Fig. 7, $C$ (velocities) and $D$ (passive eye displacement). The parameters were optimized to match the real results in Fig. 7, $A$ and $B$ (peak head acceleration $\left.=1150^{\circ} / \mathrm{s}^{2} ; \omega=84 \mathrm{rad} / \mathrm{s} ; y_{a}=0.05^{\circ} ; \tau=0.1 \mathrm{~s}\right)$. The agreement between real data and the model seems to be quite satisfactory and some features of the data are clarified. During the latency period (first $8 \mathrm{~ms}$ ), the eye movement consists entirely of the passive component. At the end of the latency period, eye velocity starts to deviate from the passive component; however, this moment cannot be unambiguously deter- mined in real data. Subsequently, eye velocity crosses the zero line to become compensatory. Obviously, this zero-crossing is delayed with respect to the real start of the active VOR, which causes an increase in the apparent latencies as measured with simple regression techniques. Furthermore, the rise in eye velocity is initially steeper than the rise in head velocity; the acceleration gain is larger than unity. This is accounted for by the contribution of the passive component. Once the passive anti-compensatory velocity has reached its maximum and starts to decrease, its acceleration becomes positive (compensatory) and will add up to the (approximately unity) acceleration generated by the active VOR. As a result, the acceleration gain at this time becomes larger than unity, as consistently observed in our real data.

To quantify these effects, we varied the anti-compensatory velocity in our model by varying the asymptotic end position $y_{a}$. The model was executed using parameters estimated from real data (Fig. 7) and the apparent gains and latencies were computed by linear regressions in exactly the same way as was done initially for the real data. A set of apparent gains and latencies thus computed from the model $(\omega=100 \mathrm{rad} / \mathrm{s} ; \tau=$ $\left.0.05 \mathrm{~s} ; y_{a}=0.0-0.1^{\circ}\right)$ is plotted in the scatter diagrams of Fig. 5, $A$ and $B$ (crosses). For latency, the model values coincide very well with the calculated regression lines. The modeled gain values rise somewhat steeper than the average real data as a function of the maximum anti-compensatory velocity, but the discrepancy is minor given the fairly schematic nature of the model.

\section{Instantaneous VOR gain}

We have shown that the acceleration gain, calculated from the slopes of the linear regressions on eye and head velocities as a function of time and reflecting an average value over a period of $\sim 40 \mathrm{~ms}$ after the latency, is larger than unity, even for far targets and in darkness (Fig. 2). Although this tendency 
was explained in Isolation and modeling of the passive eye movements as being the contribution of passive eye movements, a more profound understanding can be obtained by calculating, instead of this single gain parameter, the instantaneous gain as a continuous function of time. This can be done by comparing instantaneous eye and head velocities (or accelerations) with the appropriate time relations. Because the VOR has a latency, it is appropriate to calculate instantaneous velocity gain as the quotient of eye velocity at time $t$ and head velocity at time $t-$ latency

$$
\operatorname{Gain}(v / v)_{t}=V_{\text {eye }} / \text { head }_{t-\text { lat }}
$$

The acceleration gain is computed similarly. (This approach is analogous to systems analysis in the frequency domain, in which gain is the ratio between maximum output and input amplitudes, not the ratio between output and input at one particular moment; time shifts correspond to phase).

Figure $8 \mathrm{~A}$ shows head and eye velocities, as well as instan-
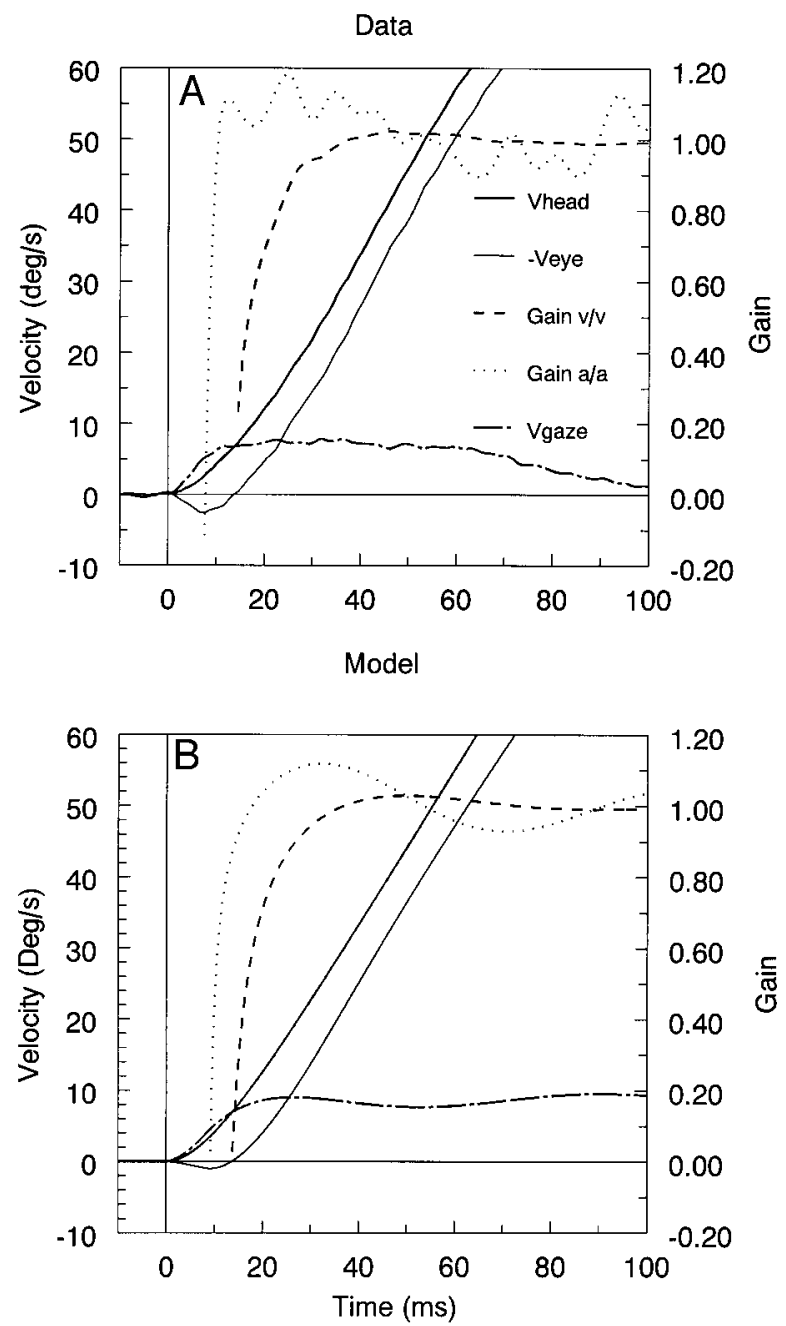

FIG. 8. A: instantaneous velocity and acceleration gains in a typical subject, calculated with due accounting for latency, i.e., $\operatorname{Gain}_{t}=V$ eye $_{t} / \operatorname{Vhead}_{(t-\text { latency })}$. Notice the steep rise above unity and subsequent oscillation of the acceleration gain in contrast to the gradual build-up of velocity gain. Notice also that gaze velocity remains substantially above zero $\left(\sim 8^{\circ} \%\right)$ for a long period (until head acceleration decreases). $B$ : simulation of these data by the model, as described in Instantaneous VOR gain. In the model simulation, gaze velocity remained high throughout because head acceleration remained constant. taneous acceleration and velocity gain, calculated for a latency of $8 \mathrm{~ms}$ for a typical subject. (To reduce noise, especially for acceleration gain, Fig. $8 A$ was prepared from a long measurement and includes 165 subsequent rightward head pulses; the target was distant and extinguished $50 \mathrm{~ms}$ before the head pulse). Obviously, velocity gain was negative as long as the eye velocity was anti-compensatory; this meaningless part was not plotted. After velocity became compensatory, velocity gain rose to a value of approximately unity. This rise was never instantaneous but took several tens of milliseconds. Acceleration gain was also initially negative but became positive as soon as the anti-compensatory eye velocity had passed its maximum, which occurred, of course, earlier than its crossing to positive values. Thus acceleration gain rose earlier than velocity gain and showed some oscillations while converging slowly toward unity. The first peak of the acceleration curve exceeded unity, which is in agreement with the average acceleration gain values of the early VOR obtained from linear regressions.

These features are perfectly duplicated by the model described in Isolation and modeling of the passive eye movements, as shown in Fig. $8 B$ (parameters, $y_{a}=0.02^{\circ} ; \omega=84$ $\mathrm{rad} / \mathrm{s} ; \tau=0.1 \mathrm{~s}$ ). The characteristic features of the gain curves are entirely determined by the occurrence of the passive, initially anti-compensatory and later oscillatory eye movements that add up to the active VOR with a constant gain of 1 . The shapes are critically affected by the correct choice of the latency and by the magnitude of the passive component. If the latency chosen is too short (or disregarded, i.e., taken to be zero, as is frequently done in the literature), gain increases much more slowly because, even with a unity gain, the latency will cause the eye velocity to remain below the simultaneous eye velocity as long as the head accelerates. On the other hand, overestimating the latency for the gain calculation (as occurred in our initial calculations from regressions) results in spurious overshoots. When the passive component is absent, either in the model or in real data, velocity and acceleration gain are identical and stable at unity throughout time after latency, when latency is correctly accounted for as in Eq. 5 .

Given the complex interactions of gain, latency, and passive components, even correctly calculated gain values are not a very transparent parameter of VOR performance. The most direct parameter of the effectiveness of the VOR is the residual retinal slip velocity, i.e., the velocity of the eye in space. Such gaze velocities are plotted as additional functions in Fig. 8, A and $B$. Gaze velocity always had the same sign as head velocity, i.e., the VOR undercompensated. As shown in Fig. $8 B$ (model simulation), a sustained head acceleration will be accompanied by a sustained gaze velocity because of the continued effect of the latency, even though gain is unity. This lag can only be overcome after head acceleration decreases and velocity levels off, as occurred in a real experiment (Fig. 8A; for the saturation effect see Fig. 1). Notice that the earliest gaze movements are even faster than the head movements because of the passive anti-compensatory response.

\section{Effect of target distance on instantaneous gain}

The effect of target conditions on the instantaneous VOR gain was studied in four subjects that were free of blinks and saccades during the first $120 \mathrm{~ms}$ after the start of head move- 
ment at $t=0$. This effect could not be adequately studied by comparing eye velocity profiles because head velocity profiles differed among our subjects. Figure $9 A$ shows averaged instantaneous VOR velocity gain for four conditions, total darkness, and a target at a distance of $220 \mathrm{~cm}$ that was switched off 50 or $500 \mathrm{~ms}$ before the head motion or left on continuously. The two movement directions and eyes were pooled; all gains were computed assuming a VOR latency of $8 \mathrm{~ms}$. The gain profiles (which were aligned at the start of head motion at $t=0$ ) were
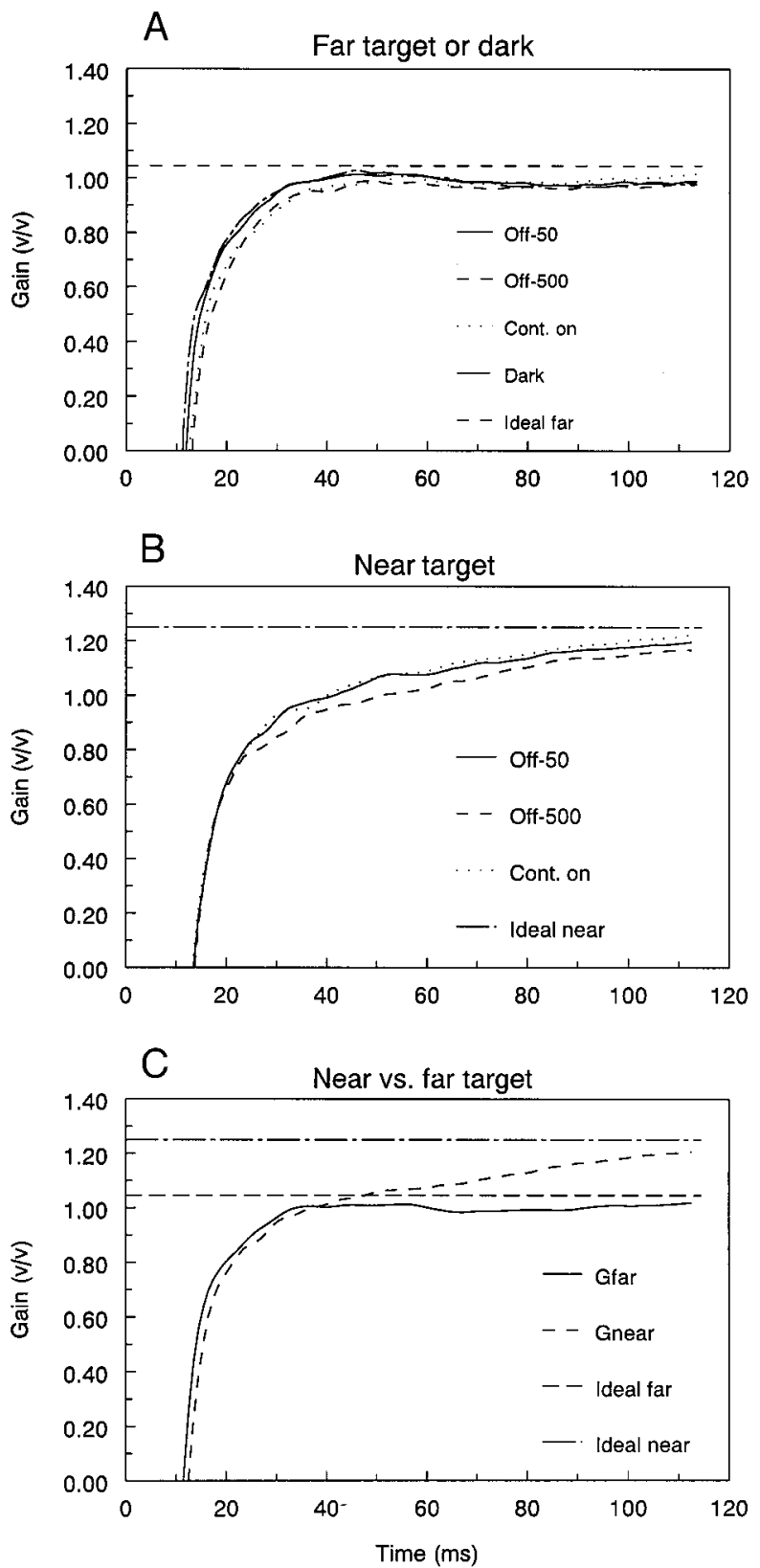

FIG. 9. A: VOR velocity gain as a function of time for distant targets, shown separately for 3 visibility conditions and darkness. Pooled values for 3 subjects and 2 directions. The VOR in darkness behaved similar to the way it did with a distant target; it made no difference whether the target was continuously visible or was extinguished shortly before head movement. Gain did not quite reach the "ideal" value. $B$ : similar graphs for near visual targets; gain rose to higher values than it did for distant targets. Once again, the results were similar for continuously visible and interrupted targets. $C$ : gain profiles compared for near and far targets (pooled data of 4 subjects). similar for all four conditions (any differences were not systematic across subjects). Velocity gain rose steeply at first (becoming positive after $\sim 12 \mathrm{~ms}$, which is when eye movement became compensatory) and later gradually, and leveled off $\sim 50 \mathrm{~ms}$ after the start of head movement. These results corroborate the fact that, in this early stage, the VOR showed identical responses in the presence of a distant target and in darkness and that short interruptions of the visibility of the target during head movement had no effect on the VOR. The overall average gain for distant targets in the interval 80-100 ms after the start of head movement was 0.998 ; variability (SD) in this period was $\sim 0.01$ for the factor time and $\sim 0.05$ for the factor subject. (Perfect compensation at this distance would require a gain of $\sim 1.045$, as shown in Fig. 9 A.)

For comparison, responses to head pulses in the presence of a near visual target (distance of $\sim 40 \mathrm{~cm}$ ) are shown, for the same subjects, in Fig. 9B. Once again, the time course of gain within the analyzed period of $\sim 120$ ms was not systematically affected by the visibility of the target (switched off 50 or 500 ms prior to the stimulus or continuously lit). The near target induced, however, a prolonged period of increasing gain as compared with a far target or darkness. After $100 \mathrm{~ms}$, mean gain was $\sim 1.2$ and had not quite reached a steady state. (Complete compensation for the near target distance would require a gain of $\sim 1.25$.) It was verified that, for all target conditions and all subjects, ocular convergence angles were appropriate for the target distance and were stable throughout the measurements at a specific distance. Thus the time of divergence between the gain for far and near targets was not temporally related to any fast change in convergence.

In Fig. 10, gain curves of these same three subjects and a fourth subject (ST, whose responses were measured with fewer variations in lighting conditions) are pooled in a different way: the average gain curves for far and near targets are shown for each of the subjects separately, and the target conditions (extinguished or not extinguished) are pooled. All subjects showed gain increasing as a function of time with eventually higher values for the near targets. The details, however, differed considerably among the subjects. Two subjects (Fig. 10, $C$ and $D$ ) showed an initially steep rise in gain, which was in agreement with the very small anti-compensatory components in these subjects. However, their VOR gain remained initially lower for near targets than it did for far targets, with a crossover occurring after only several tens of milliseconds. A third subject (Fig. 10A) showed a similar slow development of the increase in gain for near targets although gain build-up was slower in general due to a substantial anti-compensatory phase. Only one subject (Fig. 10B) showed a higher gain for the near targets from the very beginning (in combination with a large anti-compensatory component and a slow build-up of gain). Examining the different target conditions separately showed that these individual characteristics were reproducible within a subject. A complete pooling of the results for the four subjects and all of the visibility conditions for near and far targets (excluding the condition "darkness"), which shows the overall trends, is presented in Fig. 9C. The average time courses of velocity gain appeared identical for near and far targets until $\sim 40 \mathrm{~ms}$ after the start of head movement at $t=0$. After that, the time courses diverged and each of the gain curves gradually approximated the value appropriate for the target distance. 

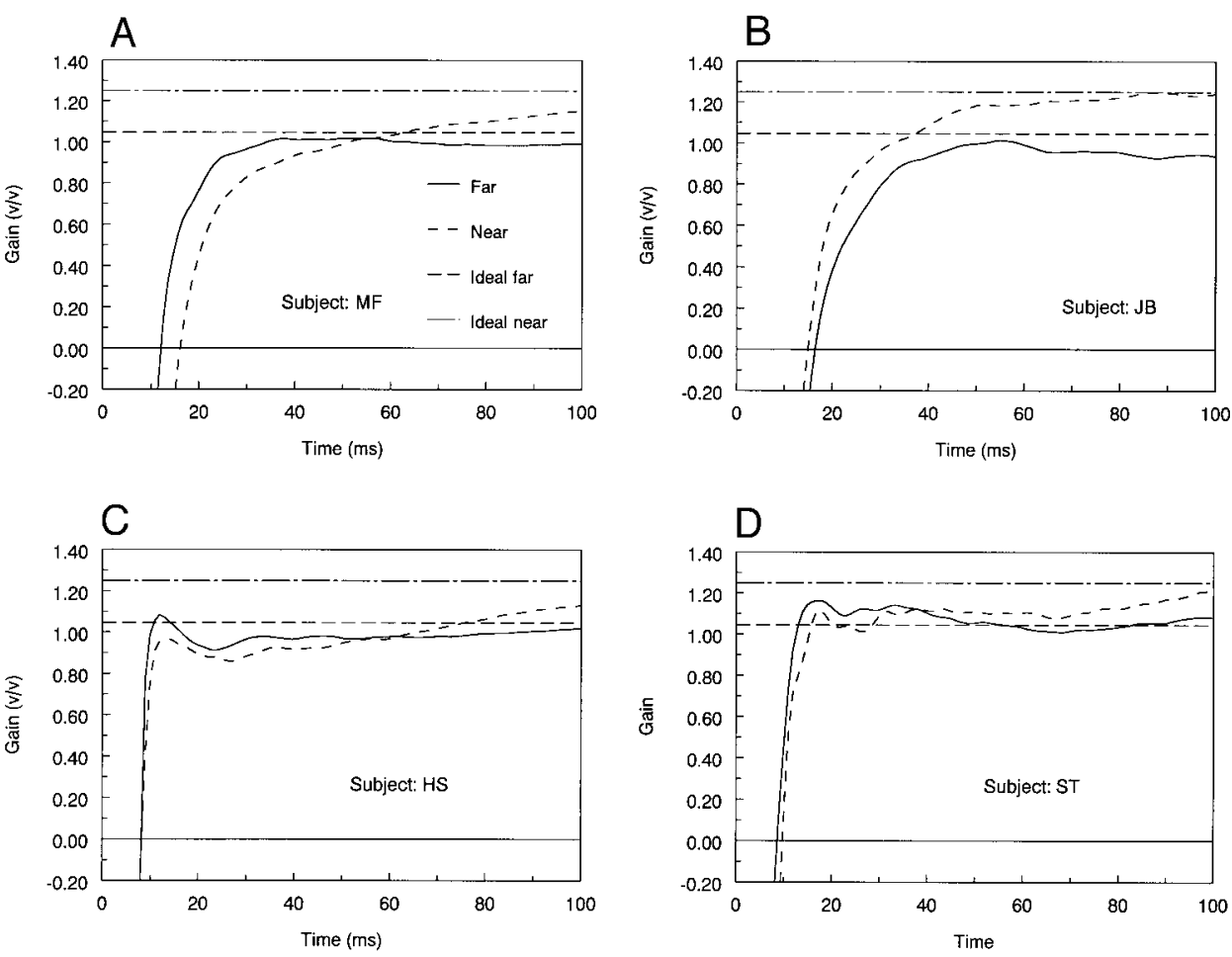

FIG. 10. VOR velocity gain as a function of time for distant and near targets. Results for 4 subjects are shown separately in $A-D$ but values for all available conditions of the visual target have been pooled.

\section{I S C U S S I O N}

The present measurements reveal a number of properties of binocular human VOR that occur during the first tens of milliseconds after a step in head acceleration of $\sim 1000^{\circ} / \mathrm{s}^{2}$. The main findings are a short latency $(\sim 8-9 \mathrm{~ms})$, a shorter latency for the contralateral eye than for the ipsilateral eye, the frequent occurrence of an anti-compensatory eye movement during the latency period, an initial acceleration gain $>1.0$ but with a gradual build-up of velocity gain, and a variable latency for an effect of target distance. We will now attempt to explain and relate these findings.

\section{Early anti-compensatory eye movements}

Surprisingly, we found that the earliest response to head acceleration was anti-compensatory in most subjects. This component had a latency of zero relative to head movement and increased during the next 7-8 $\mathrm{ms}$, after which eye acceleration in the compensatory direction originated. Given these properties, the anti-compensatory component can only be purely mechanical in origin and can only result from the assembly of eye accelerations (rotational and linear) related to the imposed head rotation. We successfully accounted for and modeled the effect on the basis of elementary physical principles related to the deformation of soft media (the orbital tissues) in a rigid vessel (the bony orbit) under the influence of pressure.

This interpretation of orbital mechanics is supported by previous studies on the ocular effects of linear accelerations. Steinbach and Lerman (1990) reported the effects of gravity on eye position in patients that were paralyzed by atracurium in preparation for a surgical procedure. An effect of gravity $(1 \mathrm{~g})$ was present in 16 of 22 patients; eye rotation (up to $5-10^{\circ}$ ) was always away from gravity, suggesting that the center of mass of the eye is behind its center of rotation. The direction of these effects corresponds to the present findings, in which leftward reactive forces resulted in a rightward passive eye movement (Fig. 6). Similarly, Bush and Miles (1996) noticed that the earliest ocular response of monkeys to a sudden free-fall (corresponding also to a change in linear acceleration by $1 \mathrm{~g}$ ) was a downward eye movement (whereas subsequent compensatory eye movements were upward). Bush and Miles (1996) interpreted this as a mechanical effect. A similar anti-compensatory effect was evident in earlier experiments on the ocular responses of monkeys from the same laboratory to translation (Schwarz and Miles 1991); the authors explicitly discussed this effect and supported its genuine nature by excluding some potential sources of artifact. No such anti-compensatory movements were mentioned by Angelaki and McHenry (1999), who performed similar translation experiments in monkeys.

In principle, it should be possible to study the dynamics of the passive effect of linear accelerations (in pure form or as a component of eccentric rotation) more directly in an isolated form in subjects with totally absent vestibular responses. In retrospect, anti-compensatory eye movements were prominent in a few patients with bilateral labyrinth defects who were investigated several years ago in our laboratory with the helmet technique (Fig. 6 in Tabak and Collewijn 1994; Fig. 4 in Tabak et al. 1997b). Halmagyi et al. (1990) detected no mechanical ocular responses in a human subject with complete bilateral vestibular neurectomy, but this absence could be due to their use of manual head rotation, during which acceleration builds up more gradually than with our helmet. Labyrinthectomy was used by Khater et al. (1993) as a control in their experiments on cat VOR, which also showed a zero-latency mechanical response that was, however, compensatory in direction. This difference in direction, compared with the reported results in humans, also emerged from experiments by Harris et al. 
(1993), who concluded, because of the effects of gravity, that the effective center of mass of the cat's eye lies in front of its center of rotation. Our present interpretation of the results of Harris et al. (1993) and Steinbach and Lerman (1990) is that effects of linear accelerations on eye orientation are actually not accounted for by the position of the center of mass of the eye as such, but by the mechanical relations between the soft orbital tissues as a whole and the surrounding bony orbital structures. These relations are likely to be different in humans and cats. Minor et al. (1999) did not observe passive eye movements in response to angular accelerations of $3000^{\circ} / \mathrm{s}^{2}$ in a monkey after bilateral labyrinthectomy, but they did not specify the position of the axis of rotation relative to the eye.

It may be possible to obtain further evidence as to what the mechanism of passive ocular responses might be by using passive rotation of normal subjects with varying positions of the rotational axis. Rotation around an axis centered on an eye should yield minimal anti-compensatory movement of that eye, whereas rotation around an axis anterior to the eye should result in inversion of the passive response to compensatory instead of anti-compensatory movement. Unfortunately, we were unable to effectively manipulate the axis of head rotation by varying the axial position of the torque applied to the helmet. The head tended to rotate around its natural axis no matter which way head stimulus was applied, and we lacked the facilities for passive whole-body rotation with comparable accelerations. Such variable axis conditions were achieved for the vertical human VOR by Viirre and Demer (1996), who applied impulsive head rotations around a horizontal axis positioned either through the centers of the eyes or $15 \mathrm{~cm}$ posterior to the eyes, and for the horizontal human VOR by Crane and Demer (1998), who varied the vertical axis position between $20 \mathrm{~cm}$ posterior and $10 \mathrm{~cm}$ anterior to the eyes. Neither of these two studies reported any early mechanical eye responses in the latency period, but neither were they mentioned as a point of attention. Although we found the anti-compensatory components to be very conspicuous in our velocity and acceleration traces, they were quite small (a few minutes of arc) in position records, which is the form in which they are recorded during experiments. They could easily be lost or escape attention if resolution at the min/arc level is slightly compromised during digital recording or subsequent data processing.

We were able to isolate the passive eye movements in favorable measurements by subtracting an "ideal" VOR (gain, 1.0 ; latency, $8 \mathrm{~ms}$ ) from the total eye movement. This revealed that the passive response to a step in eccentric rotational acceleration shows oscillations of $\sim 12-15 \mathrm{~Hz}$. This suggests a similar natural frequency of oscillation for the orbital tissues. This discovery is also important in interpreting the results of sinusoidal head oscillation in this frequency range. In previous experiments of this type (Tabak and Collewijn 1994; Tabak et al. 1997a,b), we consistently found that after a minimum at 8 $\mathrm{Hz}$ gains increased for oscillation frequencies of 14 and $20 \mathrm{~Hz}$. This trend was equally present in normal subjects and those with unilateral or bilateral labyrinth defects. Our present findings suggest that caution should be used when interpreting VOR measurements in this frequency range because they are likely to be contaminated by substantial passive contributions.

\section{Latency of the VOR}

Reliable estimates of VOR latency require 1) low-noise, high sampling frequency measurements of head and eye rotations (with insensitivity to translations); 2) transient head rotations that are well controlled in timing and magnitude; 3) absence of any spurious mechanical coupling between the stimulus and the response; and 4) suitable analysis techniques. The only recording technique that satisfies these conditions at present is the magnetic search coil technique, with search coils attached to the eye(s) and to a custom molded bite-board (or, in animals, to the bony skull). Such techniques have been applied in a number of primate and human studies (cited in the INTRODUCTION).

The present estimate of mean human VOR latency $(\sim 8-9 \mathrm{~ms})$ is consistent with our first estimate based on the helmet technique (Tabak and Collewijn 1994) and with recent measurements by Aw et al. (1996) $(7.5 \pm 2.9 \mathrm{~ms})$. Furthermore, the range of the estimated latencies (3-13 ms; see Fig. 7) is consistent with human data reported by Maas et al.(1989) (6-15 ms), Johnston and Sharpe (1994) (4-13 ms), Crane and Demer (1998) (7-10 ms), and Minor et al. (1999) $(7.3 \pm 1.5 \mathrm{~ms})$.

An intriguing new finding is the statistically robust difference in VOR latency between the eyes, the contralateral eye being $\sim 1 \mathrm{~ms}$ faster than the ipsilateral eye. This corresponds to a difference of one synaptic delay between the pathways to the lateral rectus muscle of the contralateral eye and the medial rectus muscle of the ipsilateral eye, which is in agreement with the classical description of a disynaptic pathway (vestibular afferent-medial vestibular nucleus neuron -contralateral abducens motoneuron) for the abducting eye and a trisynaptic pathway (vestibular afferent-medial vestibular nucleus neuron-internuclear neuron in the contralateral abducens nucleusipsilateral medial rectus motoneuron) for the adducting eye (for a review of these connections see Leigh and Zee 1999). The axons of the abducens internuclear neurons ascend in the contralateral medial longitudinal fasciculus (MLF). In addition, a direct pathway from medial vestibular nucleus neurons to ipsilateral medial rectus motoneurons, which runs through the ascending tract of Deiters (ATD), was described in the cat (Highstein and Baker 1978; Reisine and Highstein 1979) and in the monkey (McCrea et al. 1987). The latency difference found in the present work argues against a strong role of this ATD pathway in the human VOR. A minor role for the ATD is also suggested by experimental MLF lesions in monkeys, which cause a VOR with reduced gain with the adducting eye unable to cross the middle position (Evinger et al. 1977).

\section{VOR gain}

Gain, the ratio of the magnitudes of eye and head rotation (expressed in position, velocity, or acceleration), is generally considered to be an adequate measure of VOR performance. For distant targets it should, ideally, be close to unity to eliminate retinal image instability induced by head rotation. But the interpretation of VOR gain for transient movements is complicated by two factors: latency and mechanical transients. Confusion between gain and lag time occurs when velocity gain is calculated as the quotient of simultaneous eye and head velocities while neglecting the latency. This leads to spuriously low gain values during head acceleration because even an 
actual gain of unity will yield an eye velocity at time $t$ that matches the head velocity, not at time $t$, but at time $t$ - latency

Gain $_{\text {apparent }}=$ Veye $_{t} /$ Vhead $_{t}=[(t-$ latency $) \times a] /(t \times a)=(t-$ latency $) / t$

With a latency of $8 \mathrm{~ms}$, this leads to an apparent gain of 0 until $8 \mathrm{~ms}, 12 / 20=0.6$ after $20 \mathrm{~ms}, 32 / 40=0.8$ after $40 \mathrm{~ms}$, and $52 / 60=0.87$ after $60 \mathrm{~ms}$. In other words, gain will appear to rise slowly and will be seriously underestimated at early times (see also Tabak et al. 1997a). As an example, instantaneous VOR gain was also calculated by Crane and Demer (1998). Their graphs resemble the present ones except that their rate of rise is probably too low because they apparently did not account for latency in their gain calculations. We eliminated the effect of latency on gain by using Eq. 5. Somewhat similar corrections were applied in a few earlier studies (Aw et al.1996, Minor et al. 1999). In cases with few or no anticompensatory mechanical components, this procedure results in a steep rise of instantaneous VOR gain (Fig. 10, $C$ and $D$ ).

The presence of mechanical, initially anti-compensatory, transient eye responses also affects apparent gain, especially in the very early stage of the VOR. Although the mechanical response typically has an amplitude of only a few min/arc, its fast nature and relatively high-frequency content $(12-15 \mathrm{~Hz})$ result in substantial velocities (several \%) and high accelerations (several hundreds of $\% \mathrm{~s}^{2}$ ). The anti-compensatory movement during the latency of the VOR induces an apparent negative gain. Passive acceleration reverts to positive as soon as the anti-compensatory velocity starts to decline. Assuming a pure sinusoidal oscillation at $13 \mathrm{~Hz}$, this would occur after $\sim 19$ ms (1/4 cycle); passive velocity would become positive $19 \mathrm{~ms}$ later. This process has a cyclic nature because the passive movements are underdamped and oscillate at least as long as the period of our analysis (a little over $100 \mathrm{~ms}$ ). The passive accelerations and velocities add up to the active VOR, which starts during the first (negative) passive movement after $\sim 8$ ms. As a consequence, acceleration gain of the VOR will show oscillations around the genuine active value of $\sim 1.0$. The strongest positive effect should occur in the positive acceleration part of the first cycle of oscillation, from $\sim 19-58 \mathrm{~ms}$ after the start of the head movement. This is actually what occurs in real data (Fig. 8). This period also coincides with the period used to estimate gain from linear regressions. Therefore it is consistent that acceleration gain values determined in this way are, on average, larger than unity (Fig. 2). The regressions in the scatter diagram of Fig. $5 B$ strongly suggest that VOR acceleration gain without a passive contribution is very close to unity. It should be noted that gain values derived from regressions are not affected by the magnitude of the latency because they reflect only the ratio in the slopes of the regressions, which is independent of time. Velocity gains will also be affected, but weaker. Assuming that active VOR velocity gain, like acceleration gain, is constant and near unity for distant targets, the passive contribution should cause a maximum in the velocity gain at the peak of the second half-cycle of oscillation, i.e., about $55 \mathrm{~ms}$ after the start of head movement. This agrees with the data shown in Figs. $8 A, 9 A$, and $10, A$ and $B$. The general pattern that we found for the time course of VOR gains seems to be consistent with the observation by Minor et al. (1999) in the monkey that acceleration gain (measured early in the response) was higher (mean 1.04) than the velocity gain (mean 0.91 ) measured later when velocities had reached a plateau, although Minor's explanation was different and involved non-linearities.

\section{Effects of target conditions}

In the present experiments, visibility of the target, as well as target distance, were manipulated. The VOR was not affected by the actual visibility of a target during the transient head movements; responses were similar whether the target was continuously lit or extinguished $50 \mathrm{~ms}$ or even $500 \mathrm{~ms}$ before head rotation (Fig. 9, $A$ and $B$ ). Responses in darkness were identical to those with a distant target (Fig. 9A). This suggests that the default gain of the VOR is appropriate for distant targets. A near target caused an increase in the VOR gain, which is in agreement with the topography of the axes of rotation of eyes and head, as was reported previously (Biguer and Prablanc 1981; Blakemore and Donaghy 1980; Crane and Demer 1998; Hine and Thorn 1987; Snyder and King 1992; Snyder et al. 1992; Viirre and Demer 1996; Viirre et al. 1986). The time course of the enhancement of velocity gain by a near target varied considerably between our subjects (Fig. 10) but, at the average, a difference in gain was manifest after $\sim 40 \mathrm{~ms}$ (Fig. 9C). Higher acceleration gains for near than for far targets were also manifest in our gain values obtained from linear regressions (Fig. 2).

The few reports in the literature dealing with this aspect are not quite congruent. For monkeys, Snyder and King (1992), using accelerations of $500 \% \mathrm{~s}^{2}$, reported a modulation of the VOR by viewing distance that emerged $\sim 20-30 \mathrm{~ms}$ after the start of head rotation, i.e., $\sim 10 \mathrm{~ms}$ after the first response to head rotation. On this basis, they suggested the existence of a second, slower channel for the processing of angular head velocity signals, modified by viewing distance, in addition to a first channel that relayed only head velocity. For human subjects, Crane and Demer (1998), using accelerations of $2800^{\circ}$ / $\mathrm{s}^{2}$, found a higher VOR gain for near than for far targets throughout the response, without a delay of the expression of the distance effect such as found by Snyder and King (1992). Crane and Demer (1998) also found that a decrease in peak acceleration had nonlinear effects, among which was an increased latency for the effect of distance. Specifically, for an acceleration of $1000 \% \mathrm{~s}^{2}$ (as used in the present experiments), they found that gains became larger for near targets than they did for far targets at $\sim 32 \mathrm{~ms}$ after the start of head movement, as compared with $8 \mathrm{~ms}$ for accelerations of $2800^{\circ} / \mathrm{s}^{2}$ (their Table 2). Their average value of $32 \mathrm{~ms}$ corresponds reasonably well to the present finding of an average of $\sim 40 \mathrm{~ms}$ (Fig. 9C), but the present data suggest substantial variation among subjects of the time at which distance effects emerge.

In agreement with Viirre et al. (1986), we found a systematic difference between the acceleration gain of the two eyes, in the sense that gain was slightly higher for the eye that was closer to the target in the starting position of the head. As may be expected, the effect was statistically significant only for near targets (Fig. 2D), for which the distance of the target to the two eyes can differ substantially.

\section{REFERENCES}

AngelaKi DE AND MCHENRY MQ. Short-latency primate vestibuloocular responses during translation. J Neurophysiol 82: 1651-1654, 1999. 
Aw ST, Haslwanter T, Halmagyi GM, Curthoys IS, Yavor RA, AND Todd MJ. Three-dimensional vector analysis of the normal human vestibuloocular reflex in response to high-acceleration head rotations. I. Responses in normal subjects. J Neurophysiol 76: 4009-4020, 1996.

Biguer B and Prablanc C. Modulation of the vestibulo-ocular reflex in eye-head orientation as a function of target distance in man. In: Progress in Oculomotor Research, edited by Fuchs AF and Becker W. New York: Elsevier-North Holland, 1981, p. 525-530.

Blakemore C and Donaghy M. Co-ordination of head and eyes in the gaze changing behaviour of cats. J Physiol (Lond) 300: 317-335, 1980.

BRONSTEIN AM AND HoOD JD. The cervico-ocular reflex in normal subjects and in patients with absent vestibular function. Brain Res 373: 399-408, 1986.

Bush GA AND Miles FA. Short-latency compensatory eye movements associated with a brief period of free fall. Exp Brain Res 108: 337-340, 1996.

CARL JR AND Gellman RS. Human smooth pursuit: stimulus-dependent responses. J Neurophysiol 57: 1446-1463, 1987.

CollewiJn H. The vestibulo-ocular reflex: is it an independent subsystem? Rev Neurol (Paris) 145: 502-512, 1989.

Collewijn H, ERKelens CJ, and SteinMan RM. Voluntary binocular gazeshifts in the plane of regard: dynamics of version and vergence. Vision Res 35: 3335-3358, 1995.

Collewijn H, VAN DeR Mark F, AND Jansen TC. Precise recording of human eye movements. Vision Res 15: 447-450, 1975.

Crane BT and Demer J. Human horizontal vestibulo-ocular initiation: effects of acceleration, target distance, and unilateral deafferentiation. $J$ Neurophysiol 80: 1151-166, 1998.

Cullen KE, Belton T, and McCrea RA. A non-visual mechanism for voluntary cancellation of the vestibulo-ocular reflex. Exp Brain Res 83: 237-252, 1991

EVINGER LC, Fuchs AF, AND BAKER R. Bilateral lesions of the medial longitudinal fasciculus in monkeys: effects on the horizontal and vertical components of voluntary and vestibular induced eye movements. Exp Brain Res 28: 1-20, 1977.

Gellman RS, Carl JR, AND Miles FA. Short latency ocular-following responses in man. Vis Neurosci 5: 107-122, 1990.

Grossman GE, Leigh RJ, Abel LA, Lanska DJ, and Thurston SE. Frequency and velocity of rotational head perturbations during locomotion. Exp Brain Res 70: 470-476, 1988.

Grossman GE, Leigh RJ, Bruce EN, Huebner WP, and Lanska DJ. Performance of the human vestibuloocular reflex during locomotion. $J$ Neurophysiol 62: 264-272, 1989.

Halmagyi GM, Curthoys IS, Cremer PD, Henderson CJ, Todd MJ, StaPLES MJ, AND D'CRUZ DM. The human horizontal vestibulo-ocular reflex in response to high-acceleration stimulation before and after unilateral vestibular neurectomy. Exp Brain Res 81: 479-490, 1990.

HarRis LR, Goltz HC, and Steinbach MJ. The effect of gravity on the resting position of the cat's eye. Exp Brain Res 96: 107-116, 1993.

HighSTEIN SM. Sensory-to-motor transformations in the vestibular system. Brain Behav Evol 31: 25-33, 1988.

HIGHSTEIN SM AND BAKER R. Excitatory termination of abducens internuclear neurons on medial rectus motoneurons: relationship to syndrome of internuclear ophthalmoplegia. J Neurophysiol 41: 1647-1661, 1978.

Hine T AND Thorn F. Compensatory eye movements during active head rotation for near targets: effects of imagination, rapid head oscillation and vergence. Vision Res 27: 1639-1657, 1987.

JOHNSTON JL AND SHARPE JA. The initial vestibulo-ocular reflex and its visual enhancement and cancellation in humans. Exp Brain Res 99: 302-308, 1994.
JÜRgENS R AND MERgNER T. Interaction between cervico-ocular and vestibulo-ocular reflexes in normal adults. Exp Brain Res 77: 381-390, 1989.

Khater TT, Quinn KJ, Pena J, Baker JF, and Peterson BW. The latency of the cat vestibulo-ocular reflex before and after short- and long-term adaptation. Exp Brain Res 94: 16-32, 1993.

LeIGH RJ AND ZeE DS. The Neurology of Eye Movements (3rd ed.). New York: Oxford, 1999, p. 215-218.

LISBERGER SG. The latency of pathways containing the site of motor learning in the monkey vestibulo-ocular reflex. Science 225: 74-76, 1984.

Lisberger SG AND PAVelko TA. Vestibular signals carried by pathways subserving plasticity of the vestibulo-ocular reflex in monkeys. J Neurosci 6: 346-354, 1986

Maas EF, Huebner WP, Seidman SH, and Leigh RJ. Behavior of human horizontal vestibulo-ocular reflex in response to high-acceleration stimuli. Brain Res 499: 153-156, 1989.

McCrea RA, Strassman A, May E, and Highstein SM. Anatomical and physiological characteristics of vestibular neurons mediating the horizontal vestibulo-ocular reflex of the squirrel monkey. J Comp Neurol 264: 547570, 1987.

MinOR LB, LASKER DM, BaCKous DD, AND Hullar TE. Horizontal vestibuloocular reflex evoked by high-acceleration rotations in the squirrel monkey. I. Normal responses. J Neurophysiol 82: 1254-1270, 1999.

REISINE H AND HighSTEIN SM. The ascending tract of Deiters' conveys a head velocity signal to medial rectus motoneurons. Brain Res 170: 172-176, 1979.

RoBINSON DA. A method of measuring eye movement using a scleral search coil in a magnetic field. IEEE Trans Biomed Electr 10: 137-145, 1963.

Schwarz U AND Miles FA. Ocular responses to translation and their dependence on viewing distance. I. Motion of the observer. J Neurophysiol 66: 851-864, 1991.

SNYDER LH AND KING WM. Effect of viewing distance and location of the axis of head rotation on the monkey's vestibuloocular reflex. I. Eye movement responses. J Neurophysiol 67: 861-874, 1992.

SNYDER LH, LAWRENCE DM, AND KING WM. Changes in vestibulo-ocular reflex (VOR) anticipate changes in vergence angle in monkey. Vision Res 32: 569-575, 1992.

STEINBACH MJ AND LeRMAN J. Gravity affects resting eye position in humans. Invest Ophthal Vis Sci Suppl 31: 533, 1990.

TABAK S AND COLLEWIN H. Human vestibulo-ocular responses to rapid, helmet-driven head movements. Exp Brain Res 102: 367-378, 1994.

TABAK S AND COLLEWIJN H. Evaluation of the human vestibulo-ocular reflex at high frequencies with a helmet, driven by reactive torque. Acta Otolaryngol (Stockh) Suppl 520: 4-8, 1995.

Tabak S, Collewijn H, Boumans L.J.J.M., and Van der Steen J. Gain and delay of human vestibulo-ocular reflexes to oscillation and steps of the head by a reactive torque helmet. I. Normal subjects. Acta Otolaryngol (Stockh) 117: 785-795, 1997a

Tabak S, Collewijn H, Boumans L.J.J.M., and Van der Steen J. Gain and delay of human vestibulo-ocular reflexes to oscillation and steps of the head by a reactive torque helmet. II. Vestibular-deficient subjects. Acta Otolaryngol (Stockh) 117: 796-809, 1997b.

ViIRre E, Tweed D, MiLner K, AND Vilis T. A reexamination of the gain of the vestibuloocular reflex. J Neurophysiol 56: 439-450, 1986.

VIIRRE ES AND DEMER JL. The human vertical vestibulo-ocular reflex during combined linear and angular acceleration with near-target fixation. Exp Brain Res 112: 313-324, 1996. 